\title{
Screening for Exotic Forest Pathogens to Increase Survey Capacity Using Metagenomics
}

\author{
Émilie D. Tremblay, Marc-Olivier Duceppe, Jean A. Bérubé, Troy Kimoto, Claude Lemieux, and Guillaume J. Bilodeau† \\ First, second, and sixth authors: Canadian Food Inspection Agency (CFIA), 3851 Fallowfield Road, Ottawa, Ontario, K2H 8P9, Canada; third \\ author: Natural Resources Canada, Laurentian Forestry Centre, 1055 Du P.E.P.S. Street, P.O. Box 10380 Québec, Québec, G1V 4C7, Canada; \\ fourth author: CFIA, 4321 Still Creek Dr, Burnaby, British Columbia, V5C 6S7, Canada; and fifth author: Institut de biologie intégrative et \\ des systèmes, 1030 avenue de la Médecine, Québec, Québec, G1V 0A6, Canada.
}

Accepted for publication 17 June 2018.

\begin{abstract}
Anthropogenic activities have a major impact on the global environment. Canada's natural resources are threatened by the spread of fungal pathogens, which is facilitated by agricultural practices and international trade. Fungi are introduced to new environments and sometimes become established, in which case they can cause disease outbreaks resulting in extensive forest decline. Here, we describe how a nationwide sample collection strategy coupled to next-generation sequencing (NGS) (i.e., metagenomics) can achieve fast and comprehensive screening for exotic invasive species. This methodology can help provide guidance to phytopathology stakeholders such as regulatory agencies. Several regulated invasive species were monitored by processing field samples collected over 3 years (2013 to 2015) near high-risk areas across Canada. Fifteen sequencing runs were required on the Ion Torrent platform to process 398 samples that yielded 45 million reads. Highthroughput screening of fungal and oomycete operational taxonomic units

using customized fungi-specific ribosomal internal transcribed spacer 1 barcoded primers was performed. Likewise, Phytophthora-specific barcoded primers were used to amplify the adenosine triphosphate synthase subunit 9-nicotinamide adenine dinucleotide dehydrogenase subunit 9 spacer. Several Phytophthora spp. were detected by NGS and confirmed by species-specific quantitative polymerase chain reaction (qPCR) assays. The target species Heterobasidion annosum sensu stricto could be detected only through metagenomics. We demonstrated that screening target species using a variety of sampling techniques and NGS - the results of which were validated by qPCR - has the potential to increase survey capacity and detection sensitivity, reduce hands-on time and costs, and assist regulatory agencies to identify ports of entry. Considering that early detection and prevention are the keys in mitigating invasive species damage, our method represents a substantial asset in plant pathology management.
\end{abstract}

Among all the microorganisms, the kingdom Fungi contains the largest number of phytopathogenic species (Carris et al. 2012; Knogge 1996). Many species members of the phyla Eumycota and Oomycota newly introduced in a given country are considered important threats to plants because they can cause major harvest losses (Bilodeau et al. 2012; Mecteau et al. 2002; Vettraino et al. 2015) and ecological damage (Allen and Humble 2002; Loo 2009). Local trees and crops may not have acquired the ability to recognize these newly introduced pathogens and, therefore, their defense mechanisms may not be triggered appropriately (Raj and Dentino 2002). Although some fungi are harmless in their native environment, they can switch to a phytopathogenic state when in contact with a nonnative host with which they have not coevolved and, therefore, may cause significant ecological damage in their new environment (Bérubé and Nicolas 2015; Pirofski and Casadevall 2012; Redman et al. 2001). Typical symptoms associated with fungusand oomycete-incited diseases include root rots, cankers, wilting, and foliar and shoot damage. The genus Phytophthora stands out as

${ }^{\dagger}$ Corresponding author: G. J. Bilodeau; E-mail: guillaume.bilodeau@canada.ca

Funding: This project was funded by the CFIA-RPS project OLF-P-1304, GRDI funding (QIS and CFIA OLF-P-1411), and Genome Canada and Genome BC through a large-scale applied research program (Genome Canada grant LSARP2112) for the TAIGA collaboration project (http://taigaforesthealth.com), CFIA-RPS and CFIA-TD projects, and GRDI-CFIA mandated.

*The $\boldsymbol{e}$-Xtra logo stands for "electronic extra" and indicates that seven supplementary figures and six supplementary tables are published online.

(c) Her Majesty the Queen in Right of Canada, as represented by Canadian Food Inspection Agency, 2018. the most significant phytopathogenic oomycete because of its broad host range and its substantial impact on the agriculture and forest industries (Kamoun et al. 2015). A notorious example of such devastating oomycetes is Phytophthora infestans (Mont.) de Bary, the causal agent of potato blight, considering that it was demonstrated to be responsible for the Great Famine in Ireland between 1845 and 1852 (Goss et al. 2014; Ó'Gráda 1995).

One remarkable characteristic of most fungal and oomycete species is long-distance spore dispersal (Fry and Grunwald 2010; Tsitsigiannis et al. 2004). This feature allows for broad dissemination throughout an environment and becomes a considerable risk for tree and plant material infection following the dispersal of exotic fungal spores by wind, rain, or human-mediated distribution over kilometers or even across continents (Brown and Hovmøller 2002; Fröhlich-Nowoisky et al. 2009; Fry and Grunwald 2010; Tsitsigiannis et al. 2004; West and Kimber 2015). In addition, movement and introduction of many fungi into new areas can also be facilitated by insect vectors or by the transport of live ornamental plants, firewood, wood products, and wood packaging material. An infamous example of phytopathogenic spores vectored by the elm bark beetle is Ophiostoma novo-ulmi Brasier, the causal agent of Dutch elm disease (Allen and Humble 2002). Dutch elm disease affected millions of trees in North America after it was spread by the movement of beetle-infested firewood (Allen and Humble 2002; Negrón et al. 2005). The fact that many exotic insects are routinely intercepted in imported wood material in Canada and the United States demonstrates even more how alarming the risk associated with the introduction of such unwanted species is (Dick 1998; Haack et al. 2014; Vettraino et al. 2015) (G. S. Thurston, personal communication).

In Canada, surveys for disease vectors (e.g., wood-boring insects) and for fungal diseases are actively conducted at ports of entry and 
urban areas (Government of Canada 2011, 2013, 2014). These areas are considered at high risk for the introduction of nonindigenous species due to their exposure to increasing volumes of international commodities and proximity to potential hosts, transportation networks, and commercial activities (Bullas-Appleton et al. 2014; Eschen et al. 2015; Government of Canada 2017a; Liebhold et al. 2012). Detection surveys in these areas should represent an important component of an overall strategy for any given country to prevent the introduction and spread of invasive plant pests and to mitigate economic and environmental impacts associated (Hamelin 2012).

Many regulatory agencies currently use morphology, culturing, and established molecular assays to identify fungal and oomycete species of concern; however, these reference detection methods are considered slow, with low throughput, and often arduous (Feng et al. 2014; Goud and Termorshuizen 2003; Guarro et al. 1999; Tavanti et al. 2005; Turin et al. 2000). In addition, because many fungi can't be cultured (i.e., obligate parasites or slow-growing or complex growth media requirements), reliance on molecular methods or sequence analysis is required (Bindslev et al. 2002). To date, there are several molecular assays based on polymerase chain reactions (PCR) (e.g., real-time PCR [qPCR], conventional PCR, and isothermal amplification) and sequencing available for identification of fungal species (Aroca et al. 2008; Bilodeau et al. 2009; Capote et al. 2012; Lievens et al. 2009; Schena et al. 2006). Because molecular assays are highly specific and very sensitive when targeting multiple-copy DNA sequence regions (Gardes et al. 1991; Martin et al. 2012), they are commonly used for species screening by regulatory agencies. For example, the Canadian Food Inspection Agency (CFIA) uses qPCR assays to detect Escherichia coli O157:H7, Salmonella spp., and Listeria monocytogenes (Government of Canada 2017c,b), and the United States Department of Agriculture uses dual qPCR assays to screen for $P$. ramorum (United States Department of Agriculture 2012). However, qPCR assays only focus on a limited number of species at a time (Aroca et al. 2008; Bilodeau et al. 2009; Capote et al. 2012; Lievens et al. 2009; Schena et al. 2006).

The constant evolution of DNA analysis technologies allows researchers to study microbial communities and promotes direct use of field samples. Not only does metagenomics have the advantage of testing several samples in one run but also this method, using nextgeneration sequencing (NGS) technology, can be used to screen for a large spectrum of organisms in a given environmental sample (Fierer et al. 2007). Furthermore, NGS allows detection of lowabundance species (i.e., few sequences present) that may remain undetected by other conventional detection methods (Lindahl et al. 2013; Prigigallo et al. 2016). NGS has been applied successfully in research for fungi (Bérubé and Nicolas 2015; Leboldus et al. 2015; Malacrinò et al. 2017; Miller et al. 2016), oomycetes (Adhikari et al. 2013; Prigigallo et al. 2016), and macroinvertebrate biomonitoring in water (Carew et al. 2013; Kermarrec et al. 2014). More specifically, the Ion Torrent NGS technology has been successfully used for community studies on fungal endophytes (Kemler et al. 2013) and oomycetes (Lyon et al. 2016).

Compared with other genic regions, the internal transcribed spacer (ITS) ribosomal intergenic region-currently considered the official barcode region for fungi (Schoch et al. 2012) - is often used to identify fungi and oomycetes because its interspecific and intraspecific variation rate can differentiate more species and is more reliable for PCR purposes (Bellemain et al. 2010; Bilodeau et al. 2009; Seifert 2009; Schoch et al. 2012). However, the ITS region is not variable enough to resolve some Phytophthora spp. Using the mitochondrial DNA spacer adenosine triphosphate synthase subunit 9-nicotinamide adenine dinucleotide dehydrogenase subunit 9 (ATP9-NAD9) as an alternative marker can overcome this limitation, especially because sequences for this region are available for most of the current 123 official species (Bilodeau et al. 2014; Kang 2006; Kox et al. 2007; Martin et al. 2012; Miles et al. 2017).
This article presents a new metagenomics procedure to aid regulatory agencies in screening for fungal spores collected from air and insect vectors. Using an existing surveillance network and various sampler types, the approach innovates by analyzing the preservation fluids from CFIA's insect traps. The objectives of this proof of concept were to (i) using metagenomics, develop a new screening procedure for invasive or alien plant pathogens from air samples and insect traps near ports of entry and high risk areas in Canadian urban environments; (ii) provide guidance to phytopathology stakeholders; and (iii) compare and verify metagenomics results using previously validated qPCR assays that target fungal and oomycete species of interest.

\section{MATERIALS AND METHODS}

Target species. In 2012, the CFIA, in collaboration with the Canadian Forest Service and the Pest Management Regulatory Agency, drafted a plant pathogen research priority list that included 100 native, naturalized, and nonindigenous species. From that list, 17 phytopathogens, counting some pests regulated in Canada, were picked for this project. The target species list included species from the Phytophthora genus, ophiostomatoid fungi, and other fungi causing root rot and foliar diseases such as Bretziella fagacearum (Ceratocystis fagacearum), C. polonica, C. laricicola, Chrysomyxa abietis, Gremmeniella abietina, Geosmithia morbida, Gymnosporangium fuscum, G. yamadae, Melampsora pinitorqua, O. ulmi (i.e., O. ulmi sensu stricto), O. novo-ulmi, and Heterobasidion annosum sensu stricto. $H$. annosum sensu stricto is part of the species complex $H$. annosum sensu lato, which includes the following species: $H$. irregulare, $H$. parvivorum, $H$. annosum sensu stricto, H. abietinum, and H. occidentale (Garbelotto and Gonthier 2013; Lamarche et al. 2017). Not all target species are currently found in Canada.

Sample collection. In total, 398 environmental samples were processed. In addition, 10 mock samples inoculated with various target and closely related species and a blank sample consisting of molecular-grade water were analyzed (Caporaso et al. 2011; Ihrmark et al. 2012; Singer et al. 2016). Sampling occurred over 3 years (summer 2013,2014, and 2015) at strategically selected sites across Canada (Supplementary Table S1; Fig. 1). Sites included commercial and industrial zones, wooded urban areas, and municipal green-waste disposal facilities (Ryall et al. 2015). Four different sampling methods to survey the presence of phytopathogens were used (Supplementary Fig. S1).

First sampling method: Spore traps. The Johnson and Barnes (JB) rainfall collectors (J. L. Johnson, personal communication) consisted of a funnel appended with a filter down its throat where particles were collected on a $0.45-\mu \mathrm{m}$ filter membrane (Fisher Scientific, Waltham, MA, USA) and were processed as previously described by Barnes et al. (2006, 2009); Hambleton et al. (2007); and Szabo (2007), except that filter membranes used were made of cellulose nitrate instead. The original JB collector (funnel diameter: $44 \mathrm{~cm}$ and filter diameter: $45 \mathrm{~mm}$ ) and a smaller version, the JB mini (funnel diameter: $27 \mathrm{~cm}$ and filter diameter: $25 \mathrm{~mm}$ ), were used. Spores and rain accumulated and were then collected on the surface of the cellulose membrane filter. Collections were made once every 2 weeks in Québec and Ontario and samples were stored at $4^{\circ} \mathrm{C}$ until processed. Then, one-half of each filter was put in $1.4 \mathrm{ml}$ of Tris buffer, heated at $65^{\circ} \mathrm{C}$, sonicated at $40 \mathrm{kHz}$, and centrifuged for $2 \mathrm{~min}(10,000 \mathrm{rpm})$. The filter was then discarded and the remaining solution was centrifuged at $10,000 \mathrm{rpm}$ for $30 \mathrm{~s}$. The liquids were then dehydrated at low temperature before being extracted. The other half of the filter was kept at $-20^{\circ} \mathrm{C}$ as a backup.

Second sampling method: Preservative fluids from insect traps. Inspectors (CFIA) across Canada (British Columbia, New Brunswick, Newfoundland and Labrador, Ontario, and Québec) performed entomology surveys using Multiple Funnel Traps (Synergy Semiochemicals Corporation, Burnaby, BC, Canada), an insect collection 
device consisting of stacked funnels baited with semiochemicals (Supplementary Table S2). Preservative fluids (50 to $1,000 \mathrm{ml}$ of food-grade propylene glycol) from those insect traps' collection cups were filtered with a $0.45-\mu \mathrm{m}$ filter paper. Filter paper halves were processed as described above for JB collectors.

Third sampling method: Rotary arm sampler. The rotary arm sampler (Phytopdata Inc., Sherrington, QC, Canada) was a spinning apparatus rotating $5 \mathrm{~min}$ every hour and prepared following the protocol of Fall et al. (2015). Its two vertical metal rods (1.65 by $20 \mathrm{~mm}$ ) were dipped in a silicone grease (Versilube G697; Novagard Solutions, Cleveland, OH, USA) to facilitate spore adhesion from impacting air samples (Bérubé et al. 2017b). Samples were collected weekly in the province of Québec.

Fourth sampling method: Soil samples from agricultural fields. To initially validate the ability of our metagenomics approach to detect DNA, eight soil samples were included in our first NGS run. Six sandy loam samples were collected in the summer of 2011 from St. Rock, Alberton, PEI. They originated from yearly crop rotations of ryegrass and strawberry spread over 3 years. Samples were collected prior and after soil fumigation. We also included two peat samples collected in Vancouver, BC in 2011. Soil samples were sieved and subsampled into two 500-mg parts due to constraints of lysing matrix kit. Parts were put back together at the elution step to obtain 1-g samples.

Extraction, purification, and quantification of DNA. The $0.45-\mu \mathrm{m}$ paper filters halves from the JB collectors and the insect traps were processed as described by Barnes et al. (2009), except the FastDNA kit for soil (MP Biomedicals, Santa Ana, CA, USA) was used for DNA extraction. The same kit was used for soil samples. Previous work comparing DNA extraction methods and kits suggested that the FastDNA kit for soil was the most effective at recovering DNA from complex matrices such as environment samples when coupled with subsequent purification for PCR inhibitor removal (Bilodeau and Robideau 2014) (G. Bilodeau, unpublished). The recovery of spores and the DNA extraction from the rotary arm samples were done as described by Lamarche et al. (2017) and using the QIAmp DNA Micro kit (Qiagen, Hilden, NW, Germany). Purification of extracted DNA with magnetic beads (Bio-Nobile, Östernäsvägen, Finland) was done on all sample types to remove potential PCR inhibitors. The Qubit 2.0 fluorometer (Life Technologies, Carlsbad, CA, USA) was used to quantify DNA. A new extraction was performed for samples with a final DNA concentration lower than $0.1 \mathrm{ng} / \mu \mathrm{l}$. All DNA samples were standardized at a final concentration of $0.1 \mathrm{ng} / \mu \mathrm{l}$ for NGS library preparation.
Fusion PCR primers and design. Ion Torrent technology requires the use of fusion primers to generate tagged amplicons (Thermofisher 2012). Therefore, using the Ion Torrent's fusion primers, we designed specific PCR primer sets for fungi, oomycetes, and Phytophthora spp. This was accomplished by combining already existing primers that targeted the regions of interest (i.e., ITS1 or ATP9-NAD9) to the Ion Torrent fusion primers, which were appended with the sequencing adapters $A$ and TrP1 [P1]). These adaptors were attached to the 5' tail of the designed oligos, next to the sequence of the already existing primers used to amplify our region of interest (ITS1 or ATP9-NAD9) (Thermofisher 2012). Unique sequence ID tags were also added to the designed oligos. Doing so allowed for each PCR product (i.e., amplicon), to be tagged with a two-part barcode sequence. The first part revealed the target organism amplified-namely, eumycete or oomycete using their respective ITS1 signature and, specifically, Phytophthora spp. from which the ATP9-NAD9 region had been amplified following a positive oomycete's ITS1 amplification in order to gain specificity-and the second part indexed each sample for traceability purposes. As shown for the three indexed examples presented in Supplementary Table S3, 48 ITS1 barcodes were appended bidirectionally (96 barcodes total) for both fungi and oomycetes, and 16 forward barcodes were designed to label the Phytophthora spp. ATP9-NAD9 amplicons. More specifically, three bidirectional PCR examples are illustrated in Supplementary Fig. S2, where amplicons for the three studied regions (i.e., ITS1 fungi, ITS1 oomycete, and ATP9-NAD9 Phytophthora) and three different samples indexed are presented. Typically, the authors refer to those PCR by calling the forward (yellow) general primer (e.g., ITS1F, ITS2, and so on) name throughout the article. These newly designed barcoded primers generated amplicons from which distinguishing of the ITS1 region from fungi and oomycete amplicons and the ATP9-NAD9 region from Phytophthora amplicons was possible and, thus, for multiple field samples pooled in a single sequencing run. The Ion Amplicon Library Preparation Fusion Method was followed (Life Technologies) (Thermofisher 2012) using the alternate master mixes presented below.

PCR conditions. PCR amplifications were done with our newly designed fusion primers and sequencing adaptors A and P1 attached to generate 350- 400-bp long amplicons. In order to target general fungal species, bidirectionnal (reverse and forward directions) amplification with ITS1F (Gardes and Bruns 1993) and ITS2 (White et al. 1990) primers was done. Similarly, general oomycete

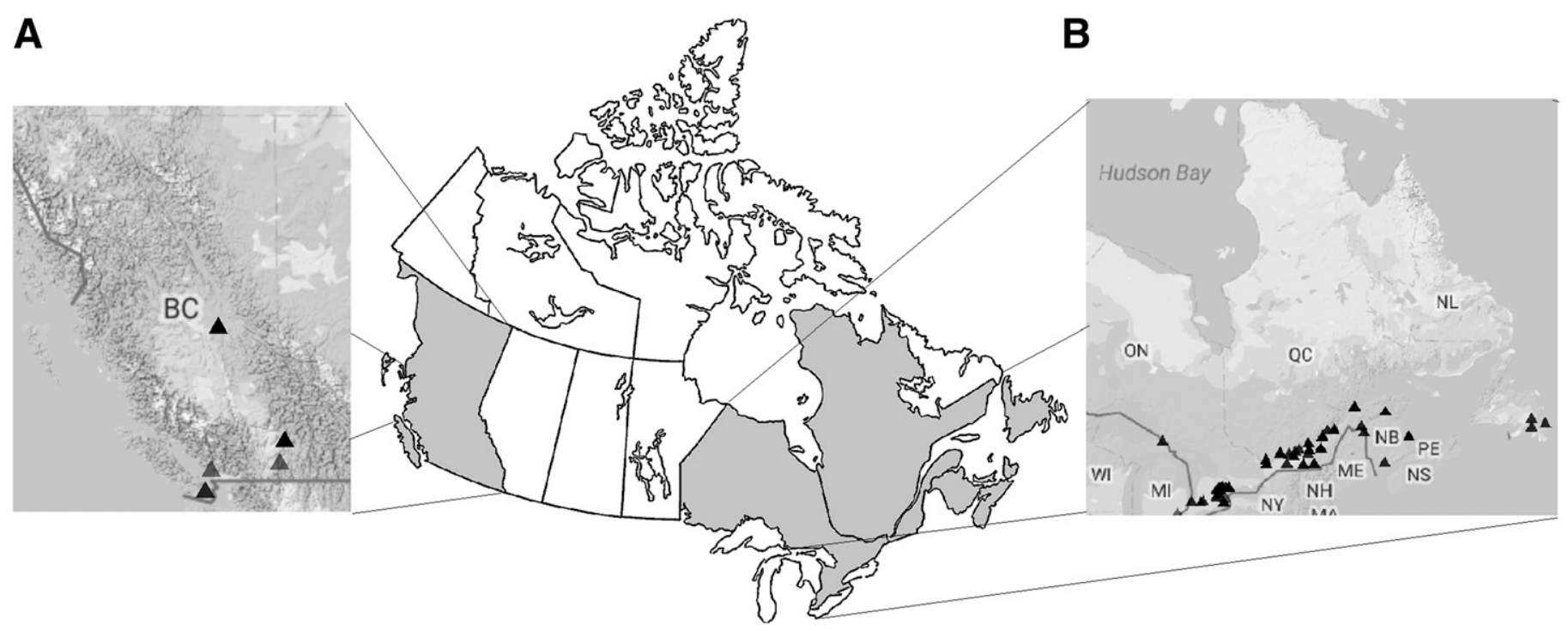

Fig. 1. Sampling sites in A, the Canadian West Coast and B, Eastern Canada. Adapted from Google Earth. 
species were targeted using bidirectional PCR amplification with fusion primers OOM-LO5.8S47 (Bilodeau et al. 2007; Man in 't Veld et al. 2002) and OOM-UP18S67 (C. A. Lévesque, personal communication). Amplification of the ATP9-NAD9 mitochondrial region from Phytophthora spp. was performed using fusion primers PhyGATP92FTail and PhyG-R6Tail (Bilodeau et al. 2014).

For fungi and oomycetes, PCR targeting the ITS1 region consisted of a $25-\mu$ PCR volume with $1 \times$ PCR buffer, $1 \mathrm{mM}$ $\mathrm{MgCl}_{2}, 0.25 \mathrm{mM} \mathrm{dNTP}, 0.50 \mathrm{mM}$ forward and reverse primers, $0.04 \mathrm{U}$ of Platinum Taq polymerase (Life Technologies), and approximately $0.1 \mathrm{ng}$ of DNA. The PCR targeting the ATP9-NAD9 region from Phytophthora spp. had a volume of $25 \mu \mathrm{l}$, including $6 \mathrm{mM} \mathrm{MgCl} 2,0.5 \mathrm{mM}$ both forward and reverse primers, $1 \times 5$ PRIME RealMasterMix Probe without ROX PCR Mix (Fisher Scientific), and approximately $0.1 \mathrm{ng}$ of DNA. Cycling conditions for ITS1 PCR were $3 \mathrm{~min}$ at $95^{\circ} \mathrm{C} ; 30$ cycles for $30 \mathrm{~s}$ at $95^{\circ} \mathrm{C}, 30 \mathrm{~s}$ at $52^{\circ} \mathrm{C}$, and $1 \mathrm{~min}$ at $72^{\circ} \mathrm{C}$ for each cycle; and $10 \mathrm{~min}$ at $72^{\circ} \mathrm{C}$. For ATP9-NAD9, PCR cycling conditions were 35 cycles with $15 \mathrm{~s}$ at $95^{\circ} \mathrm{C}$ and $90 \mathrm{~s}$ at $53^{\circ} \mathrm{C}$ for each cycle. Samples were run on a $1.5 \%$ agarose gel and visualized with a Gel Doc XR+ Gel Documentation System (Bio-Rad Laboratories, Inc., Hercules, CA, USA) to confirm DNA amplification. Smaller DNA fragments (approximately $100 \mathrm{bp}$ ) were removed by doing a purification with Agencourt AMPure XP magnetic beads at a $0.7: 1$ beads/DNA ratio (Agencourt Bioscience, Beverly, MA, USA) (Edwards 2012).

High-throughput sequencing. The Ion Universal Library Quantitation qPCR Kit (Life Technologies) was used to determine the concentration of each bidirectional barcoded sample library, which were then pooled at an equimolar concentration of $16 \mathrm{pM}$. The Ion Torrent Personal Genome Machine (Ion PGM) Template OT2 400 Kit (Life Technologies) was initially used for template preparation for runs 1 to 3 . Runs 4 to 15 used the Ion PGM Hi-Q OT2 Kit for template preparation. On the Ion PGM sequencer, enriched particles from run 13 were loaded onto a 316v2 chip which can generate 2 to 3 million reads per run. The rest of the runs were loaded onto $318 \mathrm{v} 2 \mathrm{chips}$, which can generate 4 to 5.5 million reads per run. Both the Ion PGM Sequencing $400 \mathrm{Kit}$ and the upgraded Ion PGM Hi-Q Sequencing Kit were used (Life Technologies). Samples were demultiplexed and exported (FASTQ format) using the PGM server's built-in plugins.
NGS data analysis. The bioinformatics pipeline used to analyze the Ion Torrent sequences is summarized in Figure 2. Data processing and analyses methods are further detailed below.

Raw files manipulation and filtering. FASTQ files were converted into FASTA and quality score (QUAL) files using fastqutils (Breese and Liu 2013). Sequences were trimmed based on their length and quality scores (ambiguous bases) using the trim. seqs command in Mothur (version 1.37.2) (Schloss et al. 2009) and the following parameters: minlength $=120$, maxambig $=0$, and maxhomop $=8$.

Metadata tables and operational taxonomic unit generation. Metadata tables were compiled using a custom bash script (script available upon request; metagnomics.sh) which output them in a QIIME-compatible format (version 1.7.0) (Caporaso et al. 2010). Metadata tables were then validated using the check_id_map.py script in QIIME. After this, QIIME tags were appended to the FASTA files using the add_qiime_labels.py script. The ITSx software (version 1.0.11) (Bengtsson-Palme et al. 2013) was used next to extract the ITS1 regions from fungi and oomycete samples. Operational taxonomic units (OTU) were then picked with the pick_open_reference_otus.py and pick_otu.py QIIME scripts and merged together with a parameter file. In order to avoid (i) overestimating biodiversity by generating too many OTU or (ii) omitting emerging species with a too-stringent setting, an OTU threshold of $97 \%$ was kept because it roughly corresponds to intraspecies variability level (Abdelfattah et al. 2015; DoradoMorales et al. 2015; Kemler et al. 2013; Nicolas et al. 2013; Vettraino et al. 2015). Contrary to the usual practice of discarding singletons (Henrik Nilsson et al. 2011; Mundry et al. 2012), the latter were kept to promote rare species detection.

Assignment of OTU taxonomy. The UNITE database (version 31.01.2016) (Kõljalg et al. 2005) was used to infer fungal species identity based on ITS1 sequence data (Schloss et al. 2009), whereas the National Center for Biotechnology Information (NCBI) nucleotide database was used to infer oomycete species identity based on ITS1 sequence data. A custom database of ATP9NAD9 Phytophthora spp. sequences (NCBI accessions JF771616.1 to JF772053.1 and JQ439009.1 to JQ439486.1) (Bilodeau et al. 2014) was used to infer Phytophthora spp. identity. This region offers an interesting alternative barcode region to the internal
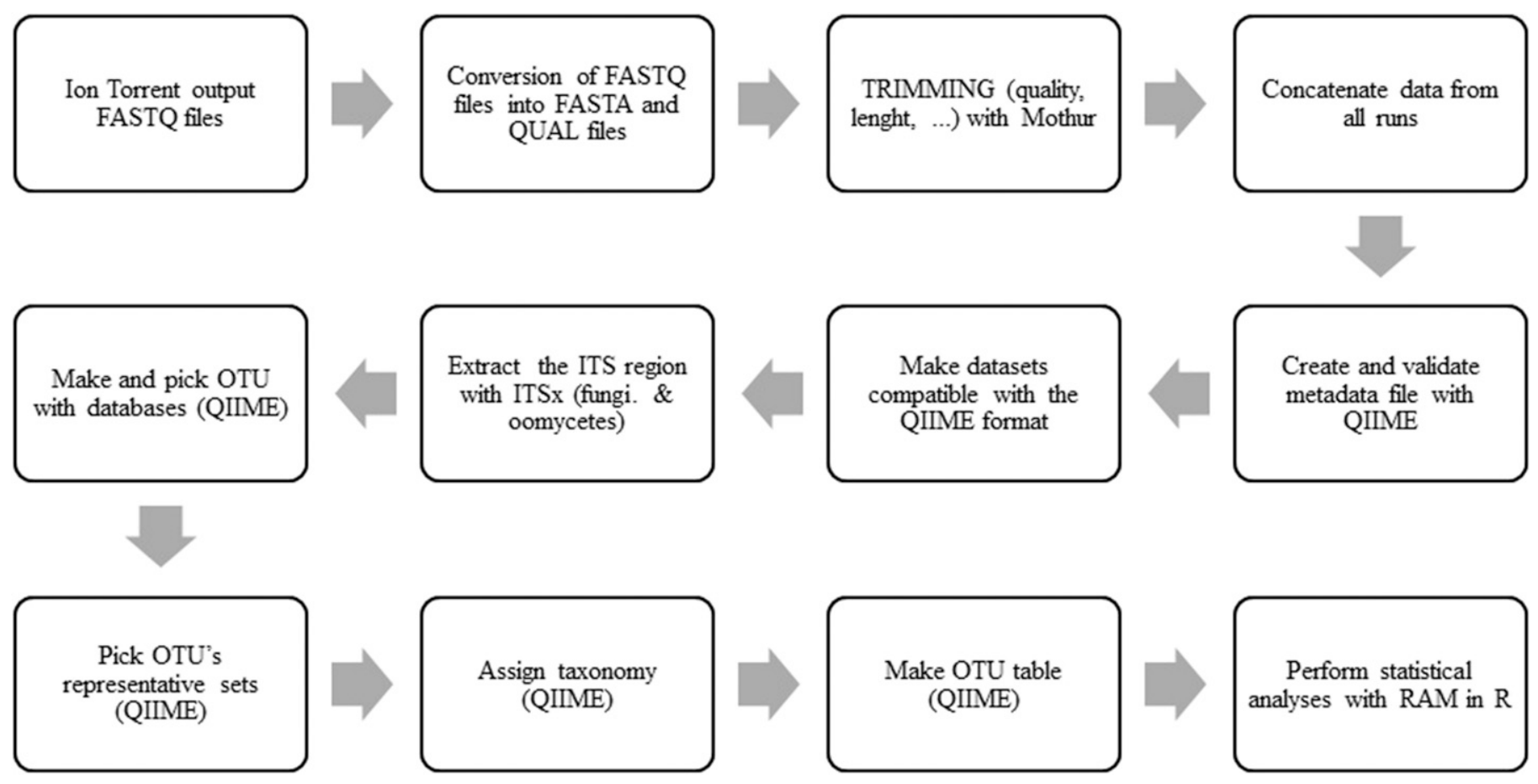

Fig. 2. Bioinformatic pipeline and tools used for next-generation sequencing analysis. 
transcribed spacer (ITS), given that our database included sequences of 140 different species or hybrids, which represents the majority of the 123 currently described species (Kang 2006). Using QIIME, representative sets of sequences were selected (pick_rep_set.py) and taxonomic information was assigned (assign_taxonomy.py) to the OTU.

OTU tables. With a modified script (parse_nonstandard_chars. py) (Walters 2015), non-ASCII characters coming from the UNITE database were removed prior to generating OTU tables (make_ otu_table.py) with QIIME. The tables, initially in the Biological Observation Matrix format, were then converted into a tabdelimited values format (TSV).

Statistics. Using the R (version 3.1.3) (R Core Team 2014) package RAM (version 1.2.1.3) (Chen et al. 2016), species richness was calculated (Cobey et al. 2013; Jost 2006; Magurran 2013). In addition, to compare the different sampling methods and to determine whether the diversity of communities was accurately represented based on the sampling and NGS protocols, rarefaction curves were done and used to normalize data and observe the sequencing depth (i.e., sufficient sampling to obtain an accurate assessment on species diversity per sample, which translates as curve saturation/plateau) according to the trap type (Abdelfattah et al. 2015; David et al. 2014; Star et al. 2013). Rarefaction curves were done with the alpha_rarefaction.py script in QIIME, where the median was set as the maximum rarefaction depth and 10 was set as the minimum value.

Species query. A custom Perl script was written to screen the species or genus targeted in this project (script available upon request; metaResultExtractor.pl). The script extracted the sample ID, sequence counts for a specific query, metadata details (city, province, collection date, lure, and trap type), and FASTA sequences, and output the data in a TSV file. A separate file with BLAST alignments performed using the NCBI (nucleotide) database was also generated by this script. All ITS1 sequences from which a target fungal genus could be inferred using the UNITE database were then realigned with the NCBI (nucleotide) database using MAFFT (version 6.240) (Katoh et al. 2002) in order to fill potential gaps (i.e., missing species) induced by UNITE. Alignments results were then visualized and edited in BioEdit (version 7.2.5) (Hall 1999) and compared with those done with UNITE.

In an attempt to resolve closely related species, phylogenetic reconstructions were performed in Geneious (version 10.1.2) (Kearse et al. 2012). Consensus trees were built with the neighborjoining method and the Jukes-Cantor genetic distance model. The resampling method consisted of 1,000 bootstraps that were used to generate a consensus tree with a $70 \%$ support threshold. Pythium vexans (oomycete) and $H$. linzhiense (fungus) were used as outgroup species or genera for the ITS1 region, whereas Plasmopara viticola was used for the ATP9-NAD9 region. FigTree (Rambaut 2016) was used to visualize the generated phylogenetic trees. Pythium vexans (outgroup) was picked because this genus's ITS sequence is so close to Phytophthora spp. that its lack of variability may not allow dissociation of Phytophthora from Pythium spp., as opposed to other, less closely related oomycetes genera. Although a species within the same genera was required to enable dissociation of the species within the species complex $H$. annosum sensu stricto, $P$. viticola was the oomycete with the most similar sequence to the genus Phytophthora among all oomycetes in the NCBI, though ATP9-NAD9 is highly variable and contains recombinant events.

Validation by species-specific qPCR assays. In order to validate NGS results, samples were also screened with speciesspecific qPCR assays. All assays were run on a ViiA 7 Real-Time PCR System (Life Technologies) and consisted of 50 cycles of $15 \mathrm{~s}$ at $95^{\circ} \mathrm{C}$ and $90 \mathrm{~s}$ at $60^{\circ} \mathrm{C}$ per cycle (Supplementary Table S4). All samples and controls were run in duplicate. Positive controls were included to validate specificity, as well as negative controls with a closely related species and no-template controls.
B. fagacearum, Ceratocystis polonica, C. laricicola, Gremmeniella abietina EU Race, and Geosmithia morbida assays. Assays consisted of a $10-\mu 1$ reaction. Each qPCR well contained $0.6 \mu \mathrm{M}$ both forward and reverse primers, $0.1 \mu \mathrm{M}$ TaqMan probe (FAM), $1 \times$ 5 PRIME RealMasterMix Probe with and without ROX PCR Mix, and approximately $0.1 \mathrm{ng}$ of DNA (Lamarche et al. 2014, 2015) (J. Lamarche, personal communication). Some primers and probes shared by our collaborators at the time were later redesigned prior to publication by Lamarche et al. (2015).

O. ulmi sensu lato, O. ulmi sensu stricto, O. novo-ulmi, O. himalulmi, and $\mathrm{H}$. annosum sensu stricto assays. The $10-\mu \mathrm{l}$ reactions consisted of $0.6 \mu \mathrm{M}$ both forward and reverse primers, $1 \mu \mathrm{M}$ TaqMan probe $(\mathrm{FAM}), 1 \times 5$ PRIME RealMasterMix Probe with and without ROX PCR Mix, and approximately $0.1 \mathrm{ng}$ of DNA (Lamarche et al. 2017) (J. Lamarche, personal communication).

Phytophthora genus and species assay. The reactions were done in a 25- $\mu$ l volume, where each reaction was filled with $6 \mathrm{mM} \mathrm{MgCl}_{2}$, $0.50 \mu \mathrm{M}$ both PhyGATP92FTail and PhyGR6Tail primers, $0.05 \mu \mathrm{M}$ both ATP9PhyG2probeR (FAM) and Pramnad9sp1F (VIC) probes, $1 \times 5$ PRIME RealMasterMix Probe with and without ROX PCR Mix, and approximately $0.1 \mathrm{ng}$ of DNA (Bilodeau et al. 2014).

Validation using mock-inoculated samples. Ten mockinoculated samples were produced to compare NGS and qPCR results under controlled conditions. Environmental sample EM14S49 was chosen to create mock samples because no target species were detected in it and to ensure proper simulation of associated sequencing errors such as per-base errors (singlenucleotide substitution) and homopolymers (Caporaso et al. 2011; Kunin et al. 2010; Salipante et al. 2014). Sample EM14S49 was collected from a JB mini spore trap in Ottawa, ON on 31 July 2014. With a concentration of approximately $0.01 \mathrm{ng} / \mu \mathrm{l}$, DNA from target species, closely related species, and other unrelated fungal species were spiked in variable amounts (i.e., 0 to $4 \mu \mathrm{l}$ ) into the mock sample (Supplementary Fig. S3), then topped up with water at a final volume of $40 \mu \mathrm{l}$. MOCS01 was a negative control (water); therefore, no additional DNA was spiked. All mock samples were subjected to both species-specific qPCR assays and NGS following the same protocol that was used on environmental samples.

\section{RESULTS}

Amplicons. Following PCR and electrophoresis done on the 398 environmental samples, bands were observed for both barcoded primer directions in $83 \%$ of samples using fungus primers and $7.8 \%$ of samples using oomycete primers. The fungus universal ITS2 barcoded primer yielded a higher frequency of amplification $(89 \%$ positive) than the fungus universal barcoded ITS1F ( $85 \%$ positive), with 355 and 339 bands obtained, respectively (Table 1). Oomycetes showed a fairly low but consistent amplification rate between the two oomycete barcoded universal primers with $8.8 \%$ (forward) and $9.8 \%$ (reverse). From the oomycete ITS1 amplicons (both barcoded directions), 36\% were positive for Phytophthora spp. following the subsequent amplification of the ATP9-NAD9 region (Table 1). Mock samples showed similar results, with an amplification rate of 90 and $100 \%$ for the universal ITS $1 F$ barcoded primer and the universal ITS2 barcoded primer, respectively (Table 2). Oomycete amplification percentages for forward and reverse barcoded primers revealed values of 83 and $100 \%$, respectively. Finally, the ATP9-NAD9 unidirectional barcoded primer set gave an amplification rate of $100 \%$ for Phytophthora spp.

NGS. In all, 13 of the 15 NGS runs performed were successful (Supplementary Table S5). Those 13 runs had an average of 77 samples and 3.5 million reads per run. Due to problems that occurred throughout the process, all samples from runs 5, 6, and 10 were sequenced again. Further analysis confirmed that the results from run 6 and the rerun samples (run 7) complemented each other because, though the data from run 6 was proportionally reduced, its contents was not compromised. In total, 45,068,371 reads and 1,151 
amplicons originating from the total 398 samples were processed. The highest number of reads obtained from a run totaled 5,980,692, whereas the lowest count was 308,794.

Fungus and oomycete diversity. Processing the NGS data through the developed bioinformatics pipeline (Fig. 2) showed that OTU and amplicon numbers (including singletons) were the highest in fungi (3,386 ITS1 OTU), compared with oomycetes (82 ITS1 OTU) and Phytophthora spp. (7 ATP9-NAD9 OTU). Comparison of the average number of sequences per OTU ranging from 18,145 to 31,381 for the organism and region studied is provided in Table 3. The blank sample (water) contained no oomycete OTU but it did contain different fungal ascomycetes and basidiomycetes species in low concentrations ( $<50$ counts): Aureobasidium sp., Candida sp., Cladosporium sp., Dothideomycetes sp., Microbotryomycetes sp., Mortierella sp., Neonectria sp., Phoma sp., Rhodotorula sp., Taphrina sp., Tremellomycetes sp., Trichocomaceae sp., and uncultured fungus.
Rarefaction curves were done on fungus and oomycete NGS samples but no rarefaction curve could be done for Phytophthora spp. samples due to the insufficient number of sequences. In the rarefaction curves generated for each sample, sequence saturation per sample was reached for all trap types. For the majority of sample types used, the optimal sequence number (saturation) in terms of number of species detected by sample versus sequencing depth was approximately 5,000 (Table 4; Supplementary Figs. S4 and S5), with the exception that there were no oomycetes detected from the rotary arm sampler. The number of species detected per trap type after normalization was approximately 500 for fungi and 35 to 40 for oomycetes (Table 4).

Target species identification. In an attempt to minimize database-dependent misidentifications, a BLAST-based custom script (metaResultExtractor.pl) that reviews all of the UNITE identification using the NCBI nucleotide database was developed. Though it confirmed that no target species were found except for the

TABLE 1. Presence or absence of amplification as detected by gel electrophoresis using barcoded polymerase chain reaction (PCR) products from 398 environmental samples from targeted organisms, and the percentages of positive reactions obtained

\begin{tabular}{|c|c|c|c|c|c|}
\hline Primers used to append barcodes ${ }^{\mathrm{a}}$ & Organism & Target region ${ }^{\mathrm{b}}$ & Positive PCR & Total PCR & Positive $(\%)^{\mathrm{c}}$ \\
\hline ITS1F-Forward & Fungi & ITS1 & 339 & 398 & 85 \\
\hline ITS1-Forward & Oomycete & ITS1 & 35 & 398 & 8.8 \\
\hline ATP9-NAD9-Forward & Phytophthora spp. & ATP9-NAD9 & 17 & 47 & 36 \\
\hline Total & $\ldots$ & $\ldots$ & 785 & 1,639 & Average: 45.7 \\
\hline
\end{tabular}

a Each PCR included a set of primers but bidirectional sequencing required one primer per direction to append the sample and organism index (i.e., barcode).

b ITS1 = internal transcribed spacer 1 and ATP9-NAD9 = adenosine triphosphate synthase subunit 9-nicotinamide adenine dinucleotide dehydrogenase subunit 9 spacer.

${ }^{c}$ Percentage of the number of positive PCR over the total number of reactions done.

TABLE 2. Presence or absence of amplification as detected by gel electrophoresis using barcoded polymerase chain reaction (PCR) products from 10 mock samples from targeted organisms, and the percentages of positive reactions associated compared with the expected results

\begin{tabular}{|c|c|c|c|c|c|}
\hline Primers used to append barcodes ${ }^{\mathrm{a}}$ & Organism & Target region ${ }^{\mathrm{b}}$ & Positive PCR & Expected positive & Positive $(\%)^{\mathrm{c}}$ \\
\hline ITS1F-Forward & Fungi & ITS1 & 9 & 10 & 90 \\
\hline ITS2-Reverse & Fungi & ITS1 & 10 & 10 & 100 \\
\hline ITS1-Forward & Oomycete & ITS1 & 6 & 6 & 100 \\
\hline ATP9-NAD9-Forward & Phytophthora spp. & ATP9-NAD9 & 6 & 6 & 100 \\
\hline Total & & & 36 & 38 & Average: 94.6 \\
\hline
\end{tabular}

a Each PCR included a set of primers but bidirectional sequencing required one primer per direction to append the sample and organism index (i.e., barcode).

b ITS1 = internal transcribed spacer 1 and ATP9-NAD9 = adenosine triphosphate synthase subunit 9-nicotinamide adenine dinucleotide dehydrogenase subunit 9 spacer.

c Percentage of the number of positive PCR - over the total number of reactions done-that were expected to be positive because the contents of inoculated samples were known.

TABLE 3. Organism sequences and operational taxonomic units (OTU) counts produced from Ion Torrent Personal Genome Machine next-generation sequencing data output

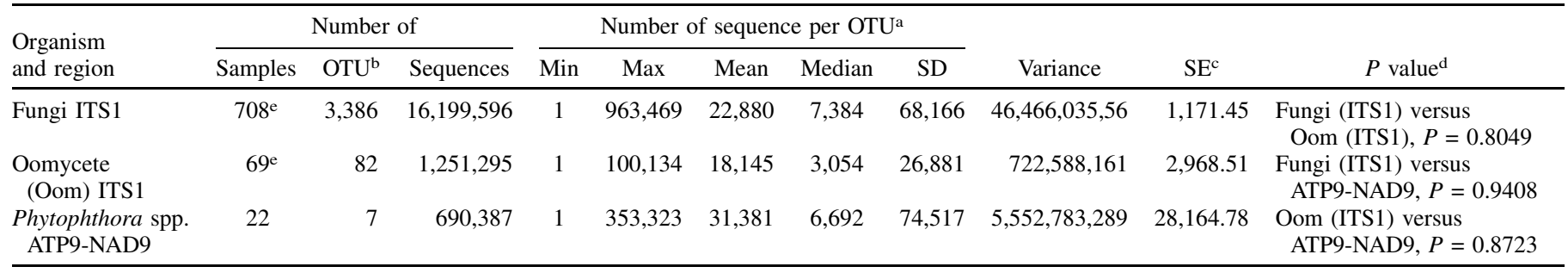

${ }^{a}$ Minimum (Min), maximum, (Max), and mean per sample library; minimum equals 1 because singletons were kept for rare species detection. SD = standard deviation.

$\mathrm{b}$ Including singletons.

c Standard error.

${ }^{\mathrm{d}}$ Calculated from an analysis of variance using the Tukey honestly significant difference posthoc test at a confidence interval of $95 \%$ (http://statpages.info/). $P$ values were used to compare OTU means between fungi (ITS1) versus oomycetes (ITS1), fungi (ITS1) versus Phytophthora spp. (ATP9-NAD9), and oomycetes (ITS1) versus Phytophthora spp. (ATP9-NAD9) in order to determine whether or not the sequence number per OTU significantly varied per region. Note that a $P$ value below 0.05 indicates a significant difference between the datasets compared.

e Sample amplicons bidirectionnally (forward and reverse) generated. 
they were distinct species. All samples containing Phytophthora material were more or less closely related to the following species: Phytophthora infestans, $P$. aff. infestans, $P$. citrophthora, P. cactorum, $P$. dreschleri, $P$. nicotianae, $P$. cactorum $\times$ nicotianae, and some Phytophthora spp. related to clades 1a, 1b, 1c, and 5 (Blair et al. 2008; Kang 2006) (F. N. Martin, J. E. Blair, and M. D. Coffey, unpublished data).

$P$. syringae was inferred based on the clusters formed after constructing a phylogenetic tree using the ATP9-NAD9 mitochondrial spacer region (Supplementary Fig. S7). Most interestingly, there were several sequences that obtained no hit from the sequence alignment with either the NCBI or the custom database. When aligned together, the BLAST hits obtained that were the closest to Phytophthora spp. were significantly different (81 to $87 \%$ identity).

Many other fungal species closely related to the targeted species were detected with the NGS workflow in environmental samples: Ceratocystis adiposa, Ceratocystis sp., C. manginecans, Geosmithia sp., H. annosum sensu lato species complex (H. irregulare and $H$. abietinum/parvivorum), Melampsora sp., O. nigrocarpum, and Ophiostoma sp. (Table 5). The majority of identified fungi belonged to phyla Ascomycota (59.7\%) and Basidiomycota (24.7\%), unidentified (11.7\%), and unclassified below kingdom (2.9\%). Although Phytophthora spp. obtained with the ITS1 amplicons equaled $6.14 \%$, most of the oomycete genera found were Peronospora spp. (27.3\%) and Pythium spp. (40\%) and a few were Hyaloperonospora spp. (1.04\%), Plasmopara spp. (0.26\%), Saprolegnia spp. (0.25\%), and Basidiophora spp. (0.32\%).

The DNA from most target species (B. fagacearum, C. fimbriata, G. morbida, Geosmithia sp., H. annosum sensu stricto, H. annosum sensu lato, O. ulmi, O. novo-ulmi, O. himal-ulmi, O. floccosum, $O$. tetropii, and $P$. ramorum) spiked into the 10 mock samples could be detected by NGS. However, some species that were added to the mock samples were not detected: C. polonica, C. laricicola, Gremmeniella abietina EU race, and G. abietina NA race.

qPCR. In the presence of the targeted DNA or positive controls, all qPCR assays performed on mock samples were positive in at least one of the two replicates, except for the assays targeting C. polonica, G. abietina, and Geosmithia morbida. These specific qPCR assays could not detect the pathogens in either mock or environmental samples.

qPCR assays performed on environmental samples screening for the pathogens B. fagacearum, C. laricicola, $H$. annosum sensu stricto, O. himal-ulmi, O. novo-ulmi, and O. ulmi sensu stricto generated no signal for any of these pathogens, with the exception of the positive controls. The qPCR duplex assay screening for Phytophthora spp. revealed several species that were different from $P$. ramorum. For the environmental samples, comparable results between the output of the Ion Torrent data and qPCR assays were observed (i.e., no detection of B. fagacearum, C. laricicola, $O$. ulmi sensu stricto, $O$. himal-ulmi, and $O$. novo-ulmi). The NGS workflow showed the presence of $H$. annosum sensu stricto in a few samples from Québec and Ontario (Table 5) despite the fact that it could not be detected by qPCR.

\section{DISCUSSION}

Pros and cons of using NGS. This proof of concept introduces a method to collect, process, and screen a high number of samples for exotic or invasive species. First, the network of samplers and the recycling of semiochemical fluids used in high-risk points of pathogen entry represent an efficient way of gathering data at a national scale in order to help diagnostics and screening efforts in the future. Plus, all the different sampling methods yielded sufficient sequencing depth per sample based on the material collected in new areas studied (Fig. 1; Table 4). These results suggest accurately represented communities. Second, the method uses custom-designed fusion primers combined with the Ion Torrent sequencing technology to monitor exotic fungi and oomycetes from multiple environmental samples simultaneously. Such a primer design approach allowed for sequencing up to 208 samples in a single run, including multiple intergenic or intergenic-flanking regions. Third, bidirectional amplification and sequencing increased sequencing coverage of amplicons, led to more robust initial species inference using databases, and yielded longer reads (350 to $400 \mathrm{bp}$ ) compared with the Illumina MiSeq pairedend technology. Fourth, the bioinformatics analysis workflow allows for processing millions of NGS reads from multiple pooled samples in just a few days. Finally, validation by speciesspecific qPCR assays strengthened the robustness of the metagenomics procedure. To our knowledge, this is the first time that fusion primers and the Ion Torrent NGS technology were used together to screen for the presence of fungi and oomycetes in air samples. The combination of the different technologies used in this new method is a fast and efficient way to evaluate information on the biota of a given location. It also has the potential to guide stakeholders for surveillance purposes such as site selection, prevention, and follow-up surveys for deeper inquiries of nonindigenous species.

This article demonstrates that, when jointly used with qPCR, NGS can be used to significantly accelerate the diagnostic process by locating regulated fungal and oomycete species directly from environmental samples and narrow down the number of downstream testing required. This represents a major asset over the current standardized methods (culturing, microscopy, qPCR from cultivated isolates, and so on) by significantly increasing throughput and drastically reducing the number of fungal isolations and culturing needed for formal identification. In addition, unlike qPCR assays, the approach developed leaves only a few samples to further assess and could detect most target and closely related species using one method rather than different protocols for each species.

The importance of mock and blank samples. DNA from several target species, congeneric species, and other unrelated species as well as one environmental sample were initially added to the 10 mock samples. The analysis of spiked samples confirmed that most pathogenic and nonpathogenic oomycete and fungal target species could be detected and identified, even in minute concentrations, by both NGS and qPCR methods. Indeed, most of the species spiked in the mock samples were persistently detected between different but comparable samples. To mimic natural conditions and add complexity to the mock samples, an environmental sample was added. The use of DNA isolates extracted from pure cultures to inoculate mock samples did not exactly emulate natural fungal and oomycete behavior but the environmental sample spiked reproduced similar conditions. Mock 1 was made with an environmental sample to ensure that its contents could be known and used as a baseline for analysis. Although fungi were detected in the blank sample (water), those were all species commonly found in either soil, water, or air or as plant endophytes. Those OTU were found in very low quantities ( $<50$ counts) and likely originated from either airborne fungal sources from the laboratory during sample processing (PCR) or were ubiquitous to the indoor environment and, thus, hard to avoid despite the controlled environment and instruments used (i.e., laminar flow hood cabinet cleaned after each use) at all times during the experiment (Cuadros-Orellana et al. 2013; Haleem Khan and Mohan Karuppayil 2012; Lindahl et al. 2013). Because none of the organisms found in the blank sample were within the same genera as our targeted species, we refuted the possibility of cross contamination with the other mock samples.

PCR outputs. In fungi, the slightly higher number of bands observed on gel when using the universal ITS2 barcoded primer versus the universal ITS1F barcoded primer may be explained by the fact that the ITS2 primer is not fungi specific, as opposed to the ITS1F primer (Table 1). These extra bands could originate from the presence of pollen and other nonfungal environmental contaminants in the samples. For oomycete PCR products, the lesser number of bands on gel observed compared with fungi are likely due 
to the fact that most oomycetes are soilborne organisms and, thus, less likely to be found in aerial samples. In addition, many more fungal species are described than oomycetes (Blackwell 2011; Hawksworth and Rossman 1997; Rossman and Palm 2006). Although the OTU numbers between oomycetes and fungi varied considerably, the comparison of the average number of sequences per OTU (Table 3) proved not to be significantly different regardless of the organism and region studied. Nevertheless, oomycete ITS1 amplicons were useful for narrowing down the number of samples to process through NGS and to target the ATP9-NAD9 region from Phytophthora spp.

NGS outputs: Fungi. Fungi produce aerial spores and are ubiquitous in the environment, which explains why all environmental samples contained fungal material, primarily species of Ascomycota and Basidiomycota (Tables 1 and 5). Because this project mainly used air and insect traps, it could also explain the type of fungal species found in the blank sample. The target species H. annosum sensu stricto was detected by NGS in environmental samples along with $H$. abietinum/parviporum and $H$. irregulare, all species of economic concern related to conifers in North America. The location where species belonging to the H. annosum sensu lato species complex were found corresponded to areas where annosus root rot was previously detected by conventional surveys near Harrington, Gatineau, and Ottawa (Laflamme and Blais 1995). Indeed, our sites were within 50 to $500 \mathrm{~km}$ from south Ontario's red pine (Pinus resinosa) plantations identified by Laflamme and Blais (1995). The detection of $H$. irregulare through our approach, though not surprising because this species is native to North America and occurs in eastern Canadian forests (Bérubé et al. 2017a; Garbelotto and Gonthier 2013; Lamarche et al. 2017), could be very useful for pest management. $H$. annosum sensu stricto and $H$. abietinum/H. parviporum are present in Europe and Asia (Garbelotto and Gonthier 2013; Lamarche et al. 2017; Woodward et al. 1998) but were also recently reported in the United States (Colorado, New Mexico, Nebraska [H. annosum sensu stricto only] and Arizona [H. annosum sensu stricto only]) (Centre for Agriculture and Biosciences International 1910; Worrall et al. 2010). For that reason, our potential spore material detection in Canada leading to formal confirmation is concordant considering the geographical proximity and, therefore, should be taken seriously as a warning to a potential increasing threat. Follow-up sampling and screening for symptomatic trees would be required to further inquire and confirm the status of $H$. annosum sensu stricto and $H$. abietinum/H. parviporum presence in Canada. It is also possible that Heterobasidion spp. previously detected in North America were misidentified for many years because the species complex concept is relatively recent. Again, such results demonstrate the importance of maintaining surveillance of this genus despite the fact that it is not among the CFIA's regulated pests.

NGS outputs: Oomycetes. Compared with fungi, only a few environmental samples contained oomycete ITS1 OTU (Tables 1, 3, and 5), and even fewer contained Phytophthora ATP9-NAD9 OTU; however, because most oomycetes, including Phytophthora spp., are soil and water organisms, this was an expected result from air and insect trap samples. NGS validated that the oomycete primers used (ITS1) were oomycete-specific because only Oomycota taxa were detected; namely, Peronospora spp., Phytophthora spp., Pythium spp., Hyaloperonospora spp., Plasmopara spp., Saprolegnia spp., and Basidiophora spp. The ITS1 amplicons generated with oomycete-specific primers revealed the presence of Phytophthora spp. in many samples but, as anticipated, no accurate identification below the genus level was possible. Notwithstanding, there were several ITS1 samples from which Phytophthora spp. such as Phytophthora syringae, Phytophthora spp. closely related to $P$. infestans, $P$. aff. infestans, $P$. citrophthora, $P$. cactorum, $P$. dreschleri, $P$. nicotianae, and $P$. cactorum $\times$ nicotianae were found. For detected species related to clades 1a, 1b, 1c, and 5 (Blair et al. 2008; Kang 2006), identification was not conclusive in most cases because sequence alignments with well-known species had lower levels of similarity (approximately 90\%). Furthermore, numerous sequences of Phytophthora ATP9-NAD9 amplicons were actually quite different ( 81 to $87 \%$ identity) compared with any other known Phytophthora sequences found in NCBI or the custom database, suggesting that they could be new species, genera, or hybrids because our samples originated from atypical oomycete environments. The low similarities observed could also be the result of biases introduced by the Ion Torrent technology itself (Quail et al. 2012), or by the lower temperature the PCR was set at. As explained by Martin et al. (2012), our knowledge of the genus Phytophthora and the number of species described is just starting to expand with new technologies and approaches such as ours but is promising to be helpful in managing and better understanding impacts of exotic species on natural resources. For instance, the highly variable and recombinant nature of the ATP9-NAD9 spacer sequences made alignments and construction of phylogenetic trees more challenging but Phytophthora spp. or clade inference was still possible in most cases.

NGS versus qPCR. Similarities were observed between NGS and qPCR results, showing the robustness of our NGS workflow. Because NGS results could be validated with species-specific qPCR assays on both environmental and mock samples, it would be very useful to obtain or develop qPCR assays for the missing targeted species such as additional Phytophthora spp. The importance of using species-specific qPCR assays in combination with NGS is highlighted by the fact that potentially new and damaging species were detected only by high-throughput sequencing. Other nonphytopathology-related scientists have actually reported results similar to ours where-just as was observed here with Heterobasidion spp.-metagenomics could detect some species which couldn't be caught by qPCR (Goodrich et al. 2016; Ladetto et al. 2014). As opposed to qPCR results, which are limited to presence or absence and quantities, NGS can also provide immediate and complex sequence information such as taxonomic evolution and species proximity.

Proper species identification when dealing with regulated pests is essential because misidentifications could have severe consequences for international trade and policies. For that reason, when discovering regulated species by NGS, it is currently necessary to complement our method with validated species-specific qPCR assays, which are considered reference standards (Adamski et al. 2014; Derveaux et al. 2010). Part of this necessity is due to the fact that metagenomics analysis relies on the use of incomplete and partially curated databases. Taxonomic profiling of environmental samples using marker genes relies on the availability of highquality databases. The fungal database UNITE was used to assign fungal taxonomy to the ITS bioinformatics workflow. Though it is a manually curated (i.e., high-quality) database, UNITE only has a limited number of sequences available. As a result, many fungal species are not present and can't be identified. Another challenge when using databases to infer the taxonomic composition of NGS sequences is the constant risk of misidentification. Because some species are genetically very similar (i.e., have very low divergence in the marker gene used), closely related species can be incorrectly identified (present or not in the database). In an attempt to avoid misidentification, a script (metaResultExtractor.pl) was developed to cross-validate the UNITE taxonomic assignments using another database (i.e., NCBI nucleotide). Analyses showed that the genus and species, when available in the database, were always inferred correctly using UNITE (data not shown). However, when species identification was not possible in UNITE, BLAST results from the NCBI (nucleotide) database produced better alignment scores due to the higher sequence number in the database. Because there is currently no curated public oomycete database available, the taxonomic assignment of oomycete ITS 1 amplicons has to rely on the NCBI (nucleotide) database. Considering that the ITS can't 
be used to distinguish Phytophthora spp., our alternative ATP9NAD9 custom database, which represents most described species, was subsequently used. By aligning the oomycete ITS1 sequences with those in the NCBI nucleotide database using BLAST, we could filter which samples had the genus Phytophthora, and then align the sequences of ATP9-NAD9 amplicons with the sequences from our custom database (ATP9-NAD9) to properly identify Phytophthora spp. However, sequence alignment differences still occurred at times. This could be due, in part, to the recent addition of sequences of newer species to the NCBI database, or because of the presence of incorrectly assigned sequences within the database. The interspecies genetic diversity may be lower than the error rate of the NGS technology, which could be another possible factor in misidentification. Despite its higher error rate $(1.71 \%)$ compared with the Illumina MiSeq (0.80\%) (Quail et al. 2012), the Ion Torrent sequencing platform was chosen because, at the time, it could produce longer reads (up to $400 \mathrm{bp}$, the actual size of the ITS 1 region in fungi and oomycetes and the ATP9-NAD9 region in Phytophthora spp.), an appreciable asset when doing molecular identifications. Comparatively, the Illumina MiSeq platform only yielded sequence data up to $150 \mathrm{bp}$ when the project was initiated in 2013 (Quail et al. 2012).

Three species-C. polonica (mocks 2, 3, 9, and 10), Gremmeniella abietina EU race (mocks 2, 3, 5, and 6), and Geosmithia morbida (mocks 2 and 3)-were not detected by either qPCR or NGS from the spiked mock samples. We could likely not detect them by NGS using the ITS1 region because it lacks sufficient variation to resolve those specific species (Lamarche et al. 2014, 2015). As for the qPCR assays, although the $\beta$-tubulin and RNA polymerase B II genes - those alternate genes targeted by the qPCR assays - can achieve species resolution due to their higher variation rates (Lamarche et al. 2014, 2015), they also failed to detect species, most likely because they are single-copy genes and, therefore, less sensitive (Aguileta et al. 2008; Ayliffe et al. 2001; Bellemain et al. 2010; Schmitt et al. 2009). In comparison, the ITS region is found in multiple copies in fungal and oomycete genomes (up to 251 copies/ cell) (Aguileta et al. 2008; Ayliffe et al. 2001; Bellemain et al. 2010; Schmitt et al. 2009), a tremendous advantage when working with minute quantities of DNA and with mixed samples. This advantage alone overcomes the fact that some species can't be resolved with the ITS or other regions.

In this article, we presented a fast and high-throughput NGSbased method to monitor and evaluate fungal phytopathogenic material in environmental samples originating from air and insect traps. Validated with qPCR assays, NGS data analyzed with a powerful customized bioinformatics pipeline outputs taxonomic composition of samples which can be used as a highly relevant tool for regulatory agencies, organizations, and countries. Although limited by database quality, we clearly showed that our NGS method can simultaneously and efficiently process large numbers of field-collected samples and has the potential for screening almost all fungal and oomycete species, including previously uncharacterized species. Additional testing, culture validation, Koch's postulates, and follow-up surveys in the field to seek for symptomatic trees shall still be performed, especially in situations where regulatory actions are necessary.

More importantly, this new method was able to detect spores of Heterobasidion and Phytophthora spp. in a specific environment, despite the fact that it could not provide information about the extent of establishment or a disease's status. Used as a screening tool, this protocol identified high-risk areas (Gatineau, QC; Ottawa, ON; and Vancouver, BC) for species of interest and allowed for analysis on a national scale. Although the ITS1 is unable to resolve certain species, it can be applied within a broader surveillance program or at specific locations to pinpoint samples that require further investigation. Validated species-specific assays (qPCR), when available, are still required to confirm the presence of species from NGS data.

\section{ACKNOWLEDGMENTS}

We thank the anonymous reviewers of our manuscript for their helpful comments; J. Dubé for the rotary-arm spore sample processing; our field collaborators N. Bérubé, J.-F. Pépin, J. Turcotte, R. Ouellet, M. Lepage, P. St-Laurent, R. Morisset, and A. Bates for hosting and operating the rotary-arm spore collectors; R. Neville, L. Gagné, E. Bullas-Appleton, the CFIA inspection staff, and R. Wilson for providing insect trap samples; P. Tanguay and R. Hamelin for providing samples and DNA isolates; M. Newton and H. Sayed for offering support with sample processing; A. Abdelfattah, E. Giroux, W. Chen, I. Kandalaft, G. Nicolas, and C. Lowe for helping with the bioinformatics analysis; J. Lamarche, P. Tanguay, and R. Hamelin for sharing qPCR assays; and L. Bernier for his support and advice.

\section{LITERATURE CITED}

Abdelfattah, A., Nicosia, M. G. L. D., Cacciola, S. O., Droby, S., and Schena, L. 2015. Metabarcoding analysis of fungal diversity in the phyllosphere and carposphere of olive (Olea europaea). PLoS One 10:e0131069.

Adamski, M. G., Gumann, P., and Baird, A. E. 2014. A method for quantitative analysis of standard and high-throughput qPCR expression data based on input sample quantity. PLoS One 9:e103917.

Adhikari, B. N., Hamilton, J. P., Zerillo, M. M., Tisserat, N., Lévesque, C. A., and Buell, C. R. 2013. Comparative genomics reveals insight into virulence strategies of plant pathogenic oomycetes. PLoS One 8:e75072.

Aguileta, G., Marthey, S., Chiapello, H., Lebrun, M.-H., Rodolphe, F., Fournier, E., Gendrault-Jacquemard, A., and Giraud, T. 2008. Assessing the performance of single-copy genes for recovering robust phylogenies. Syst. Biol. 57:613-627.

Allen, E., and Humble, L. 2002. Nonindigenous species introductions: A threat to Canada's forests and forest economy. Can. J. Plant Pathol. 24:103-110.

Aroca, A., Raposo, R., and Lunello, P. 2008. A biomarker for the identification of four Phaeoacremonium species using the $\beta$-tubulin gene as the target sequence. Appl. Microbiol. Biotechnol. 80:1131-1140.

Ayliffe, M. A., Dodds, P. N., and Lawrence, G. J. 2001. Characterisation of a $\beta$-tubulin gene from Melampsora lini and comparison of fungal $\beta$-tubulin genes. Mycol. Res. 105:818-826.

Barnes, C. W., Szabo, L. J., and Bowersox, V. 2009. Identifying and quantifying Phakopsora pachyrhizi spores in rain. Phytopathology 99:328-338.

Barnes, C. W., Szabo, L. J., Johnson, J. L., Nguyen, K. P., Floyd, C. M., and Kurle, J. E. 2006. Detection of Phakopsora pachyrhizi DNA in rain using qPCR and a portable rain collector. (Abstr.) Phytopathology 96:S9.

Bellemain, E., Carlsen, T., Brochmann, C., Coissac, E., Taberlet, P., and Kauserud, H. 2010. ITS as an environmental DNA barcode for fungi: An in silico approach reveals potential PCR biases. BMC Microbiol. 10:189.

Bengtsson-Palme, J., Ryberg, M., Hartmann, M., Branco, S., Wang, Z., Godhe, A., Wit, P., Sánchez-García, M., Ebersberger, I., and Sousa, F. 2013. Improved software detection and extraction of ITS1 and ITS2 from ribosomal ITS sequences of fungi and other eukaryotes for analysis of environmental sequencing data. Methods Ecol. Evol. 4:914-919.

Bérubé, J. A., Dubé, J., and Potvin, A. 2017a. Incidence of Heterobasidion irregulare aerial basidiospores at different locations in southern Quebec. Can. J. Plant Pathol. 40:34-38.

Bérubé, J. A., and Nicolas, G. G. 2015. Alien fungal species on asymptomatic live woody plant material imported into Canada. Can. J. Plant Pathol. 37:67-81.

Bérubé, J. A., Potvin, A., and Stewart, D. 2017b. Importance of local and longdistance Heterobasidion irregulare aerial basidiospore dispersal for future infection centres in thinned red pine plantations in Quebec. For. Chron. 93: 241-245.

Bilodeau, G., Lévesque, C., De Cock, A., Duchaine, C., Brière, S., Uribe, P., Martin, F., and Hamelin, R. 2007. Molecular detection of Phytophthora ramorum by real-time polymerase chain reaction using TaqMan, SYBR Green, and molecular beacons. Phytopathology 97:632-642.

Bilodeau, G., Pelletier, G., Pelletier, F., Lévesque, C., and Hamelin, R. 2009. Multiplex real-time polymerase chain reaction (PCR) for detection of Phytophthora ramorum, the causal agent of sudden oak death. Can. J. Plant Pathol. 31:195-210.

Bilodeau, G. J., Koike, S. T., Uribe, P., and Martin, F. N. 2012. Development of an assay for rapid detection and quantification of Verticillium dahliae in soil. Phytopathology 102:331-343.

Bilodeau, G. J., Martin, F. N., Coffey, M. D., and Blomquist, C. L. 2014. Development of a multiplex assay for genus- and species-specific detection of Phytophthora based on differences in mitochondrial gene order. Phytopathology 104:733-748.

Bilodeau, G. J., and Robideau, G. P. 2014. Optimization of nucleic acid extraction from field and bulk samples for sensitive direct detection of plant pests. (Abstr.) Phytopathology 104:S3.14. 
Bindslev, L., Oliver, R. P., and Johansen, B. 2002. In situ PCR for detection and identification of fungal species. Mycol. Res. 106:277-279.

Blackwell, M. 2011. The Fungi: 1, 2, 3.. 5.1 million species? Am. J. Bot. 98: 426-438.

Blair, J. E., Coffey, M. D., Park, S.-Y., Geiser, D. M., and Kang, S. 2008. A multi-locus phylogeny for Phytophthora utilizing markers derived from complete genome sequences. Fungal Genet. Biol. 45:266-277.

Breese, M. R., and Liu, Y. 2013. NGSUtils: A software suite for analyzing and manipulating next-generation sequencing datasets. Bioinformatics 29: 494-496.

Brown, J. K., and Hovmøller, M. S. 2002. Aerial dispersal of pathogens on the global and continental scales and its impact on plant disease. Science 297: 537-541.

Bullas-Appleton, E., Kimoto, T., and Turgeon, J. J. 2014. Discovery of Trichoferus campestris (Coleoptera: Cerambycidae) in Ontario, Canada and first host record in North America. Can. Entomol. 146:111-116.

Caporaso, J. G., Kuczynski, J., Stombaugh, J., Bittinger, K., Bushman, F. D., Costello, E. K., Fierer, N., Pena, A. G., Goodrich, J. K., and Gordon, J. I. 2010. QIIME allows analysis of high-throughput community sequencing data. Nat. Methods 7:335-336.

Caporaso, J. G., Lauber, C. L., Walters, W. A., Berg-Lyons, D., Lozupone, C. A., Turnbaugh, P. J., Fierer, N., and Knight, R. 2011. Global patterns of $16 \mathrm{~S}$ rRNA diversity at a depth of millions of sequences per sample. Proc. Natl. Inst. Sci. USA 108:4516-4522.

Capote, N., Aguado, A., Pastrana, A. M., and Sánchez-Torres, P. 2012. Molecular tools for detection of plant pathogenic fungi and fungicide resistance. Pages 151-202 in: Plant Pathology. C. J. Cumagun, ed. Online publication. InTech

Carew, M. E., Pettigrove, V. J., Metzeling, L., and Hoffmann, A. A. 2013. Environmental monitoring using next generation sequencing: Rapid identification of macroinvertebrate bioindicator species. Front. Zool. 10:45

Carris, L. M., Little, C. R., and Stiles, C. M. 2012. Introduction to Fungi. Online publication. Plant Health Instruct.

Centre for Agriculture and Biosciences International. 1910. CABI. Online publication. https://www.cabi.org

Chen, W., Simpson, J., and Levesque, C. 2016. RAM: R for AmpliconSequencing-Based Microbial-Ecology $\mathrm{R}$ package, version 1.2.1.3. Online publication. https://cran.r-project.org/web/packages/RAM/index.html

Cobey, S. W., Tarpy, D. R., and Woyke, J. 2013. Standard methods for instrumental insemination of Apis mellifera queens. J. Apic. Res. 52:1-18.

Cuadros-Orellana, S., Leite, L. R., Smith, A., Medeiros, J. D., Badotti, F., Fonseca, P. L., Vaz, A. B., Oliveira, G., and Góes-Neto, A. 2013. Assessment of fungal diversity in the environment using metagenomics: A decade in review. Fungal Genomics Biol. 3:110.

David, V., Terrat, S., Herzine, K., Claisse, O., Rousseaux, S., Tourdot-Maréchal, R., Masneuf-Pomarede, I., Ranjard, L., and Alexandre, H. 2014. Highthroughput sequencing of amplicons for monitoring yeast biodiversity in must and during alcoholic fermentation. J. Ind. Microbiol. Biotechnol. 41:811-821.

Derveaux, S., Vandesompele, J., and Hellemans, J. 2010. How to do successful gene expression analysis using real-time PCR. Methods 50:227-230.

Dick, M. 1998. Pine pitch canker-the threat to New Zealand. N. Z. For. 42: 30-34.

Dorado-Morales, P., Vilanova, C., Garay, C. P., Martí, J. M., and Porcar, M. 2015. Unveiling bacterial interactions through multidimensional scaling and dynamics modeling. Sci. Rep. 5: Article 18396.

Edwards, D. 2012. PCR Purification: AMPure and Simple. Online publication. http://www.keatslab.org/blog/pcrpurificationampureandsimple

Eschen, R., Britton, K., Brockerhoff, E., Burgess, T., Dalley, V., Epanchin-Niell, R., Gupta, K., Hardy, G., Huang, Y., and Kenis, M. 2015. International variation in phytosanitary legislation and regulations governing importation of plants for planting. Environ. Sci. Policy 51:228-237.

Fall, M. L., Tremblay, D. M., Gobeil-Richard, M., Couillard, J., Rocheleau, H., Van der Heyden, H., Lévesque, C. A., Beaulieu, C., and Carisse, O. 2015. Infection efficiency of four Phytophthora infestans clonal lineages and DNA-based quantification of sporangia. PLoS One 10:e0136312.

Feng, X., Wu, Z., Ling, B., Pan, S., Liao, W., Pan, W., and Yao, Z. 2014. Identification and differentiation of Candida parapsilosis complex species by use of exon-primed intron-crossing PCR. J. Clin. Microbiol. 52:17581761.

Fierer, N., Breitbart, M., Nulton, J., Salamon, P., Lozupone, C., Jones, R., Robeson, M., Edwards, R. A., Felts, B., and Rayhawk, S. 2007. Metagenomic and small-subunit rRNA analyses reveal the genetic diversity of bacteria, archaea, fungi, and viruses in soil. Appl. Environ. Microbiol. 73: 7059-7066.

Fröhlich-Nowoisky, J., Pickersgill, D. A., Després, V. R., and Pöschl, U. 2009. High diversity of fungi in air particulate matter. Proc. Natl. Inst. Sci. USA 106:12814-12819.

Fry, W. E., and Grunwald, N. J. 2010. Introduction to Oomycetes. Online publication. Plant Health Instruct.
Garbelotto, M., and Gonthier, P. 2013. Biology, epidemiology, and control of Heterobasidion species worldwide. Annu. Rev. Phytopathol. 51:39-59.

Gardes, M., and Bruns, T. D. 1993. ITS primers with enhanced specificity for basidiomycetes - application to the identification of mycorrhizae and rusts. Mol. Ecol. 2:113-118

Gardes, M., White, T. J., Fortin, J. A., Bruns, T. D., and Taylor, J. W. 1991. Identification of indigenous and introduced symbiotic fungi in ectomycorrhizae by amplification of nuclear and mitochondrial ribosomal DNA. Can. J. Bot. 69:180-190.

Goodrich, D., Tao, X., Bohrer, C., Lonczak, A., Xing, T., Zimmerman, R., Zhan, Y., Scott, R. T., Jr., and Treff, N. R. 2016. A randomized and blinded comparison of qPCR and NGS-based detection of aneuploidy in a cell line mixture model of blastocyst biopsy mosaicism. J. Assist. Reprod. Genet. 33:1473-1480.

Goss, E. M., Tabima, J. F., Cooke, D. E., Restrepo, S., Fry, W. E., Forbes, G. A., Fieland, V. J., Cardenas, M., and Grünwald, N. J. 2014. The Irish potato famine pathogen Phytophthora infestans originated in central Mexico rather than the Andes. Proc. Natl. Inst. Sci. USA 111:8791-8796.

Goud, J., and Termorshuizen, A. 2003. Quality of methods to quantify microsclerotia of Verticillium dahliae in soil. Eur. J. Plant Pathol. 109:523-534.

Government of Canada. 2011. D-99-03: Phytosanitary Measures to Prevent the Entry of Oak Wilt Disease (Ceratocystis fagacearum (Bretz) Hunt) from the Continental United States. Online publication. http://www.inspection.gc.ca/ plants/plant-pests-invasive-species/plant-pest-surveillance/eng/1344466499681/ $1344466638872 \#$ p3

Government of Canada. 2013. D-01-01: Phytosanitary Requirements to Prevent the Entry and Spread of Phytophthora ramorum. Online publication. http://www.inspection.gc.ca/plants/plant-pests-invasive-species/directives/ horticulture/d-01-01/eng/1323825108375/1323825214385

Government of Canada. 2014. D-03-08: Phytosanitary Requirements to Prevent the Introduction Into and Spread Within Canada of the Emerald Ash Borer, Agrilus planipennis (Fairmaire). Online publication. http://www.inspection.gc.ca/plants/plant-pests-invasive-species/directives/forestry/d-0308/eng/1323821135864/1323821347324

Government of Canada. 2017a. Plant Pest Surveillance. Online publication. http://www.inspection.gc.ca/plants/plant-pests-invasive-species/plant-pestsurveillance/eng/1344466499681/1344466638872\#p3

Government of Canada. 2017b. Laboratory Procedures for the Microbiological Analysis of Foods-Volume 3: The Compendium of Analytical Methods. Online publication. https://www.canada.ca/en/health-canada/services/foodnutrition/research-programs-analytical-methods/analytical-methods/compendiummethods/laboratory-procedures-microbiological-analysis-foods-compendiumanalytical-methods.html?wbdisable=true

Government of Canada. 2017c. Annex D: List of Canadian microbiological screening and cultural methods for Salmonella, Listeria monocytogenes, Escherichia coli O157:H5 recognized as equivalent by the FSIS. Online publication. http://www.inspection.gc.ca/food/meat-and-poultry-products/ manual-of-procedures/chapter-11/united-states-of-america/annex-d/eng/ $1369764984299 / 1369765125333$

Guarro, J., Gené, J., and Stchigel, A. M. 1999. Developments in fungal taxonomy. Clin. Microbiol. Rev. 12:454-500.

Haack, R. A., Britton, K. O., Brockerhoff, E. G., Cavey, J. F., Garrett, L. J., Kimberley, M., Lowenstein, F., Nuding, A., Olson, L. J., and Turner, J. 2014. Effectiveness of the International Phytosanitary Standard ISPM No. 15 on reducing wood borer infestation rates in wood packaging material entering the United States. PLoS One 9:e96611.

Haleem Khan, A. A., and Mohan Karuppayil, S. 2012. Fungal pollution of indoor environments and its management. Saudi J. Biol. Sci. 19:405-426.

Hall, T. A. 1999. BioEdit: A user-friendly biological sequence alignment editor and analysis program for Windows 95/98/NT. Nucleic Acids Symp. Ser. 41:95-98.

Hambleton, S., Tenuta, A., Anderson, T., Tropiano, R., Bergeron, J., and Van Herk, C. 2007. Asian soybean rust monitoring program pays off in 2007 with first detections in Canada. In: Proc. 2007 Natl. Soybean Rust Symp. Online publication. Plant Management Network. http://www.plantmanagementnetwork.org/infocenter/topic/soybeanrust/2007/posters/14.asp

Hamelin, R. C. 2012. Contributions of genomics to forest pathology. Can. J. Plant Pathol. 34:20-28.

Hawksworth, D. L., and Rossman, A. Y. 1997. Where are all the undescribed fungi? Phytopathology 87:888-891.

Henrik Nilsson, R., Tedersoo, L., Lindahl, B. D., Kjøller, R., Carlsen, T., Quince, C., Abarenkov, K., Pennanen, T., Stenlid, J., and Bruns, T. 2011. Towards standardization of the description and publication of next-generation sequencing datasets of fungal communities. New Phytol. 191:314-318.

Ihrmark, K., Bödeker, I. T., Cruz-Martinez, K., Friberg, H., Kubartova, A., Schenck, J., Strid, Y., Stenlid, J., Brandström-Durling, M., and Clemmensen, K. E. 2012. New primers to amplify the fungal ITS2 region-evaluation by 454-sequencing of artificial and natural communities. FEMS Microbiol. Ecol. 82:666-677. 
Jost, L. 2006. Entropy and diversity. Oikos 113:363-375.

Kamoun, S., Furzer, O., Jones, J. D. G., Judelson, H. S., Ali, G. S., Dalio, R. J. D., Roy, S. G., Schena, L., Zambounis, A., Panabières, F., Cahill, D., Ruocco, M., Figueiredo, A., Chen, X.-R., Hulvey, J., Stam, R., Lamour, K., Gijzen, M., Tyler, B. M., and Grunwald, N. J. 2015. The Top 10 oomycete pathogens in molecular plant pathology. Mol. Plant Pathol. 16:413-434.

Kang, S. 2006. The Phytophthora database Project. Online publication. http:// www.phytophthoradb.org/

Katoh, K., Misawa, K., Kuma, K.-i., and Miyata, T. 2002. MAFFT: A novel method for rapid multiple sequence alignment based on fast Fourier transform. Nucleic Acids Res. 30:3059-3066.

Kearse, M., Moir, R., Wilson, A., Stones-Havas, S., Cheung, M., Sturrock, S., Buxton, S., Cooper, A., Markowitz, S., and Duran, C. 2012. Geneious Basic: An integrated and extendable desktop software platform for the organization and analysis of sequence data. Bioinformatics 28:1647-1649.

Kemler, M., Garnas, J., Wingfield, M. J., Gryzenhout, M., Pillay, K.-A., and Slippers, B. 2013. Ion torrent PGM as tool for fungal community analysis: A case study of endophytes in Eucalyptus grandis reveals high taxonomic diversity. PLoS One 8:e81718

Kermarrec, L., Franc, A., Rimet, F., Chaumeil, P., Frigerio, J.-M., Humbert, J.-F., and Bouchez, A. 2014. A next-generation sequencing approach to river biomonitoring using benthic diatoms. Freshw. Sci. 33:349-363.

Knogge, W. 1996. Fungal infection of plants. Plant Cell 8:1711-1722.

Kõljalg, U., Larsson, K.-H., Abarenkov, K., Nilsson, R. H., Alexander, I. J., Eberhardt, U., Erland, S., Høiland, K., Kjøller, R., Larsson, E., Pennanen, T., Sen, R., Taylor, A. F. S., Tedersoo, L., Vrålstad, T., and Ursing, B. M. 2005. UNITE: A database providing web-based methods for the molecular identification of ectomycorrhizal fungi. New Phytol. 166:1063-1068.

Kox, L. F. F., van Brouwershaven, I. R., van de Vossenberg, B., van den Beld, H. E., Bonants, P. J. M., and de Gruyter, J. 2007. Diagnostic values and utility of immunological, morphological, and molecular methods for in planta detection of Phytophthora ramorum. Phytopathology 97:1119-1129.

Kunin, V., Engelbrektson, A., Ochman, H., and Hugenholtz, P. 2010. Wrinkles in the rare biosphere: Pyrosequencing errors can lead to artificial inflation of diversity estimates. Environ. Microbiol. 12:118-123.

Ladetto, M., Brüggemann, M., Monitillo, L., Ferrero, S., Pepin, F., Drandi, D., Barbero, D., Palumbo, A., Passera, R., and Boccadoro, M. 2014. Nextgeneration sequencing and real-time quantitative PCR for minimal residual disease detection in B-cell disorders. Leukemia 28:1299-1307.

Laflamme, G., and Blais, R. 1995. Détection du Heterobasidion annosum au Québec. Phytoprotection 76:39-43.

Lamarche, J., Potvin, A., Pelletier, G., Stewart, D., Feau, N., Alayon, D. I., Dale, A. L., Coelho, A., Uzunovic, A., and Bilodeau, G. J. 2015. Molecular Detection of 10 of the Most Unwanted Alien Forest Pathogens in Canada Using Real-Time PCR. PLoS One 10:e0134265.

Lamarche, J., Potvin, A., Stewart, D., Blais, M., Pelletier, G., Shamoun, S., Hamelin, R., and Tanguay, P. 2017. Real-time PCR assays for the detection of Heterobasidion irregulare, $H$. occidentale, $H$. annosum sensu stricto and the Heterobasidion annosum complex. For. Pathol. 47:e12321.

Lamarche, J., Stewart, D., Pelletier, G., Hamelin, R. C., and Tanguay, P. 2014. Real-time PCR detection and discrimination of the Ceratocystis coerulescens complex and of the fungal species from the Ceratocystis polonica complex validated on pure cultures and bark beetle vectors. Can. J. For. Res. 44:1103-1111.

Leboldus, J. M., Kinzer, K., Richards, J., Ya, Z., Yan, C., Friesen, T. L., and Brueggeman, R. 2015. Genotype-by-sequencing of the plant-pathogenic fungi Pyrenophora teres and Sphaerulina musiva utilizing Ion Torrent sequence technology. Mol. Plant Pathol. 16:623-632.

Liebhold, A. M., Brockerhoff, E. G., Garrett, L. J., Parke, J. L., and Britton, K. O. 2012. Live plant imports: The major pathway for forest insect and pathogen invasions of the US.. Front. Ecol. Environ. 10:135-143.

Lievens, B., Van Baarlen, P., Verreth, C., Van Kerckhove, S., Rep, M., and Thomma, B. P. 2009. Evolutionary relationships between Fusarium oxysporum $\mathrm{f}$. sp. lycopersici and F. oxysporum $\mathrm{f}$. sp. radicis-lycopersici isolates inferred from mating type, elongation factor- $1 \alpha$ and exopolygalacturonase sequences. Mycol. Res. 113:1181-1191.

Lindahl, B. D., Nilsson, R. H., Tedersoo, L., Abarenkov, K., Carlsen, T., Kjøller, R., Kõljalg, U., Pennanen, T., Rosendahl, S., and Stenlid, J. 2013. Fungal community analysis by high-throughput sequencing of amplified markers-a user's guide. New Phytol. 199:288-299.

Loo, J. A. 2009. Ecological impacts of non-indigenous invasive fungi as forest pathogens. Biol. Invasions 11:81-96.

Lyon, R., Correll, J., Feng, C., Bluhm, B., Shrestha, S., Shi, A., and Lamour, K. 2016. Population Structure of Peronospora effusa in the Southwestern United States. PLoS One 11:e0148385.

Man in 't Veld, W. A., de Cock, A. W. A. M., Ilieva, E., and Lévesque, C. A. 2002. Gene flow analysis of Phytophthora porri reveals a new species: Phytophthora brassicae sp. nov. Eur. J. Plant Pathol. 108:51-62.

Magurran, A. E. 2013. Measuring Biological Diversity. John Wiley \& Sons, Oxford, UK
Malacrinò, A., Rassati, D., Schena, L., Mehzabin, R., Battisti, A., and Palmeri, V. 2017. Fungal communities associated with bark and ambrosia beetles trapped at international harbours. Fungal Ecol. 28:44-52.

Martin, F. N., Abad, Z. G., Balci, Y., and Ivors, K. 2012. Identification and detection of Phytophthora: Reviewing our progress, identifying our needs. Plant Dis. 96:1080-1103.

Mecteau, M. R., Joseph, A., and Tweddell, R. J. 2002. Effect of organic and inorganic salts on the growth and development of Fusarium sambucinum, a causal agent of potato dry rot. Mycol. Res. 106:688-696.

Miles, T. D., Martin, F. N., Robideau, G., Bilodeau, G., and Coffey, M. 2017. Systematic development of Phytophthora species-specific mitochondrial diagnostic markers for economically important members of the genus. Plant Dis. 101:1162-1170

Miller, K. E., Hopkins, K., Inward, D. J., and Vogler, A. P. 2016. Metabarcoding of fungal communities associated with bark beetles.. Ecol. Evol. 6:1590-1600.

Mundry, M., Bornberg-Bauer, E., Sammeth, M., and Feulner, P. G. 2012. Evaluating characteristics of de novo assembly software on 454 transcriptome data: A simulation approach. PLoS One 7:e31410.

Negrón, J. F., Witcosky, J. J., Cain, R. J., LaBonte, J. R., Duerr, D. A., McElwey, S. J., Lee, J. C., and Seybold, S. J. 2005. The banded elm bark beetle: A new threat to the elms in North America. Am. Entomol. (Lanham Md.) 51:84-94.

Nicolas, G. G., Ponchart, J., and Berube, J. A. 2013. Détection de nouveaux champignons pathogènes forestiers sur des arbres urbains par séquençage à haut débit en criblage in silico. ACFAS, Université Laval, Québec, Canada.

Ó'Gráda, C. 1995. The Great Irish Famine. Cambridge University Press.

Pirofski, L., and Casadevall, A. 2012. Q\&A: What is a pathogen? A question that begs the point. BMC Biol. 10:6.

Prigigallo, M. I., Abdelfattah, A., Cacciola, S. O., Faedda, R., Sanzani, S. M., Cooke, D. E., and Schena, L. 2016. Metabarcoding Analysis of Phytophthora Diversity Using Genus-Specific Primers and 454 Pyrosequencing. Phytopathology 106:305-313.

Quail, M., Smith, M., Coupland, P., Otto, T. D., Harris, S. R., Connor, T. R., Bertoni, A., Swerdlow, H. P., and Gu, Y. 2012. A tale of three next generation sequencing platforms: Comparison of Ion Torrent, Pacific Biosciences and Illumina MiSeq sequencers. BMC Genomics 13:341.

R Core Team. 2014. R: A Language and Environment for Statistical Computing. R Foundation for Statistical Computing, Vienna.

Raj, P. A., and Dentino, A. R. 2002. Current status of defensins and their role in innate and adaptive immunity. FEMS Microbiol. Lett. 206:9-18.

Rambaut, A. 2016. FigTree v1.4.3 2006-2016. Tree Figure Drawing Tool. Online publication. Institute of Evolutionary Biology, University of Edinburgh. https://vcru.wisc.edu/simonlab/bioinformatics/programs/figtree/ README.txt

Redman, R. S., Dunigan, D. D., and Rodriguez, R. J. 2001. Fungal symbiosis from mutualism to parasitism: Who controls the outcome, host or invader? New Phytol. 151:705-716.

Rossman, A. Y., and Palm, M. 2006. Why are Phytophthora and other Oomycota not true Fungi? Online publication. The American Phytopathological Society. https://www.apsnet.org/edcenter/intropp/PathogenGroups/ Pages/Oomycetes.aspx

Ryall, K., Silk, P., Webster, R. P., Gutowski, J. M., Meng, Q., Li, Y., Gao, W., Fidgen, J., Kimoto, T., and Scarr, T. 2015. Further evidence that monochamol is attractive to Monochamus (Coleoptera: Cerambycidae) species, with attraction synergised by host plant volatiles and bark beetle (Coleoptera: Curculionidae) pheromones. Can. Entomol. 147:564-579.

Salipante, S. J., Kawashima, T., Rosenthal, C., Hoogestraat, D. R., Cummings, L. A., Sengupta, D. J., Harkins, T. T., Cookson, B. T., and Hoffman, N. G. 2014. Performance comparison of Illumina and Ion Torrent next-generation sequencing platforms for $16 \mathrm{~S}$ rRNA-based bacterial community profiling. Appl. Environ. Microbiol. 80:7583-7591.

Schena, L., Hughes, K. J., and Cooke, D. E. 2006. Detection and quantification of Phytophthora ramorum, P. kernoviae, P. citricola and P. quercina in symptomatic leaves by multiplex real-time PCR. Mol. Plant Pathol. 7: 365-379.

Schloss, P. D., Westcott, S. L., Ryabin, T., Hall, J. R., Hartmann, M., Hollister, E. B., Lesniewski, R. A., Oakley, B. B., Parks, D. H., and Robinson, C. J. 2009. Introducing mothur: Open-source, platform-independent, communitysupported software for describing and comparing microbial communities. Appl. Environ. Microbiol. 75:7537-7541.

Schmitt, I., Crespo, A., Divakar, P., Fankhauser, J., Herman-Sackett, E., Kalb, K., Nelsen, M., Nelson, N., Rivas-Plata, E., and Shimp, A. 2009. New primers for promising single-copy genes in fungal phylogenetics and systematics. Persoonia 23:35-40.

Schoch, C. L., Seifert, K. A., Huhndorf, S., Robert, V., Spouge, J. L., Levesque, C. A., Chen, W., Bolchacova, E., Voigt, K., and Crous, P. W. 2012. Nuclear ribosomal internal transcribed spacer (ITS) region as a universal DNA barcode marker for Fungi. Proc. Natl. Inst. Sci. USA 109:6241-6246. 
Seifert, K. A. 2009. Progress towards DNA barcoding of fungi. Mol. Ecol. Resour. 9:83-89.

Singer, E., Andreopoulos, B., Bowers, R. M., Lee, J., Deshpande, S., Chiniquy, J., Ciobanu, D., Klenk, H.-P., Zane, M., and Daum, C. 2016. Next generation sequencing data of a defined microbial mock community. Sci. Data 3:160081.

Star, B., Haverkamp, T. H., Jentoft, S., and Jakobsen, K. S. 2013. Next generation sequencing shows high variation of the intestinal microbial species composition in Atlantic cod caught at a single location. BMC Microbiol. 13:248.

Szabo, L. J. 2007. Spore trapping: Technologies and results from 2007. In: Natl. Soybean Rust Symp. The American Phytopathological Society, Louisville, KY.

Tavanti, A., Davidson, A. D., Gow, N. A., Maiden, M. C., and Odds, F. C. 2005. Candida orthopsilosis and Candida metapsilosis spp. nov. to replace Candida parapsilosis groups II and III. J. Clin. Microbiol. 43:284-292.

Thermofisher. 2012. Ion Amplicon Library Preparation (Fusion Method). Online publication. http://tools.thermofisher.com/content/sfs/manuals/ 4468326_IonAmpliconLibraryPrep_FusionMethod_UG.pdf

Tsitsigiannis, D. I., Zarnowski, R., and Keller, N. P. 2004. The lipid body protein, PpoA, coordinates sexual and asexual sporulation in Aspergillus nidulans. J. Biol. Chem. 279:11344-11353.

Turin, L., Riva, F., Galbiati, G., and Cainelli, T. 2000. Fast, simple and highly sensitive double-rounded polymerase chain reaction assay to detect medically relevant fungi in dermatological specimens. Eur. J. Clin. Invest. 30:511-518.

United States Department of Agriculture. 2012. Shared document list for P. ramorum-related diagnostics. Online publication. https://www.aphis.usda.gov/ plant_health/plant_pest_info/pram/downloads/pdf_files/DiagnosticsTable.pdf

Vettraino, A. M., Roques, A., Yart, A., Fan, J.-T., and Sun, J.-H. 2015. Sentinel trees as a tool to forecast invasions of alien plant pathogens. PLoS One 10:e0120571.

Walters, T. 2015. parse_nonstandard_chars.py - removes any characters that are not ASCII 127. Online publication. https://github.com/alk224/akutilsv1.1.1/blob/master/parse_nonstandard_chars.py

West, J. S., and Kimber, R. B. E. 2015. Innovations in air sampling to detect plant pathogens. Ann. Appl. Biol. 166:4-17.

White, T. J., Bruns, T., Lee, S., and Taylor, J. W. 1990. Amplification and direct sequencing of fungal ribosomal RNA genes for phylogenetics. Pages 315-322 in: PCR Protocols: A Guide to Methods and Applications. M. A. Innis, D. H. Gelfand, J. J. Sninsky, and T. J. White, eds. Academic Press, New York.

Woodward, S., Stenlid, J., Karjalainen, R., and Huttermann, A. 1998. Pages xi-xii in: Heterobasidion annosum: Biology, Ecology, Impact and Control. University of Michigan Press, Ann Arbor.

Worrall, J. J., Harrington, T. C., Blodgett, J. T., Conklin, D. A., and Fairweather, M. L. 2010. Heterobasidion annosum and H. parviporum in the southern Rocky Mountains and adjoining states. Plant Dis. 94:115-118. 